\title{
THE UNIQUENESS OF THE ONE-DIMENSIONAL PARABOSON FIELD
}

\author{
BY
}

\author{
STEVEN ROBBINS
}

\begin{abstract}
A paraboson analog of the one-dimensional boson field is discussed and a uniqueness result similar to a result of Putnam is obtained. It is shown that the paraboson operators must be unbounded.
\end{abstract}

Introduction. Green first introduced the parafermion and paraboson field algebras in 1953 [2]. Much is known about the representation of the parafermion field operators but there has been little mathematical work concerning representations in the paraboson case due to the unbounded nature of the operators. No bounded relations corresponding to the Weyl relations for bosons have been found so the paraboson operators must be treated in unbounded form. In one dimension the paraboson relations reduce to the single relation

$$
C^{*} C^{2}-C^{2} C^{*}=2 C,
$$

where $C$ is a creation operator and its adjoint $C^{*}$ is an annihilation operator. This relation cannot be taken literally but must be given a suitable interpretation. We will prove a uniqueness result parallel to the ones proved by Putnam and Tillmann for bosons. As a corollary to the proof it will be shown that there are no bounded operators satisfying the paraboson relations. This is clear when the polar decomposition of $C$ yields a self-adjoint operator with discrete spectrum, an assumption which is usually made but is not necessarily satisfied.

Uniqueness of the paraboson field. Let $C$ be a closed, densely defined linear operator on a Hilbert space $K$ satisfying

$$
C^{*} C=C C^{*}+I \text {. }
$$

Putnam [5] proved that $C^{*} C$ then has purely discrete spectrum consisting of the nonnegative integers. Tillmann $[8$, p. 263] also showed that $\{C, K\}$ is unitarily equivalent to a direct sum of one-dimensional free boson fields. See also [6, Theorem 4.4.1].

Received by the editors March 21, 1974 and, in revised form, June 18, 1974.

AMS (MOS) subject classifications (1970). Primary 47B25, 47B47; Secondary 81 A20, 81 A57.

Key words and phrases. Parastatistics, paraboson, quantum field, positive energy. 
We will extend this result to the paraboson case but first we will need an analog of the one-dimensional free boson field.

Definition. A one-dimensional free paraboson field of order $p>0$ is a pair $\{C, K\}$, where $K$ is a Hilbert space with orthonormal basis $\left\{e_{j}: j=0,1,2\right.$, $\ldots\}$ and $C$ is the closed, densely defined operator on $K$ such that $C e_{j}=\gamma_{j} e_{j+1}$ where

$$
\gamma_{j}= \begin{cases}\sqrt{j+1}, & j \text { even } \\ \sqrt{j+p}, & j \text { odd }\end{cases}
$$

Note that when $p=1, \gamma_{j}=\sqrt{j+1}$ and this gives the free boson field. $p$ can be any nonnegative number and, unlike the parafermion case, need not be an integer.

We are now able to state our main result.

THEOREM. Let $C$ be a closed, densely defined linear operator on a Hilbert space $K$ such that $C C^{*}$ and $C^{*} C$ commute,

$$
C^{*} C^{2}=C^{2} C^{*}+2 C,
$$

and either $C C^{*}+C^{*} C$ has discrete spectrum or $\{C, K\}$ is completely reducible. Then both of the latter two conditions hold and $\{C, K\}$ is unitarily equivalent to a direct sum of free paraboson fields.

The relation (1) implies that $C C^{*}$ and $C^{*} C$ commute while (2) only implies that formally their commutator vanishes. We will need that the spectral projections of $C C^{*}$ and $C^{*} C$ commute (which can easily be checked in the case of the free paraboson field) so we are forced to assume the commutativity directly. It is not sufficient to assume that the commutator of $C C^{*}$ and $C^{*} C$ vanishes on a dense invariant domain. See, for example, [4, pp. 603-606]. In the boson case the complete reducibility is automatic while for parabosons it is not. This is because the operator $n=1 / 2\left(C C^{*}+C^{*} C\right)$ which plays the part of the number operator may have continuous spectrum in the paraboson case when the representation is reducible.

The theorem will be proved in two parts. We first handle the discrete spectrum case and then show that when $n$ has a partially continuous spectrum the representation is reducible.

Let $n=1 / 2\left(C C^{*}+C^{*} C\right)$. $n$ is self-adjoint and nonnegative since $C C^{*}$ and $C^{*} C$ are nonnegative commuting self-adjoint operators. Let $n=\Omega \lambda E(d \lambda)$ be the spectral resolution of $n$. Define

$$
D=\{w \in K: E(\Delta) w=w, \text { for some bounded interval } \Delta\} .
$$


$D$ is dense in $K$ and $n$ is essentially self-adjoint on $D$. Since $C C^{*}$ and $C^{*} C$ commute with $n, D$ is invariant under these operators. Thus $D$ is a subset of the domains of $C, C^{*}, C C^{*}, C^{*} C, C^{2} C^{*}, C C^{*} C$ and $C^{*} C C^{*}$. (2) implies that $D$ is also a subset of the domain of $C^{*} C^{2}$. From these domain conditions and (2) it follows that for $w \in D$,

$$
\left[C^{*}, C^{2}\right] w=2 C w, \quad[n, C] w=C w .
$$

Let $\sigma_{d}(n)$ be the discrete spectrum of $n$ and assume $\sigma_{d}(n) \neq \varnothing$. Choose $q \in \sigma_{d}(n)$ such that $q-1 \notin \sigma_{d}(n)$. Choose $v \in K$ such that $n v=q v, v \neq 0$. Then $v \in D$ and $n C v=C(n+1) v=(q+1) C v$, so $C v \in D$. Similarly, $C^{k} v \in D$ and $n C^{k} v=(q+k) C^{k} v$. Let $M$ be the set of finite linear combinations of $C^{k} v$. $M \subseteq D$ so $M \subseteq \operatorname{Dom}(C) \cap \operatorname{Dom}\left(C^{*}\right)$. Clearly $C M \subseteq M$. We will show that $C^{*} M$ $\subseteq M$. To see this let $w \in D$.

$$
\begin{aligned}
\left\langle n w, C^{*} v\right\rangle & =\langle C n w, v\rangle=\langle(n-1) C w, v\rangle \\
& =\langle C w,(n-1) v\rangle=\langle C w,(q-1) v\rangle \\
& =\left\langle w,(q-1) C^{*} v\right\rangle .
\end{aligned}
$$

Since $n$ is essentially self-adjoint on $D, C^{*} v \in \operatorname{Dom}(n)$ and $n C^{*} v=(q-1) C^{*} v$. This implies that $C^{*} v=0$ since $(q-1) \notin \sigma_{d}(n)$.

If $k>1$,

$$
C^{*} C v=C^{*} C v+C C^{*} v=2 n v=2 q v \in M .
$$

$$
\begin{aligned}
& C^{*} C^{k} v=C^{*} C^{2}\left(C^{k-2} v\right)=C^{2} C^{*} C^{k-2} v+2 C^{k-1} v, \\
& C^{*} C^{k} v=C^{2 j} C^{*} C^{k-2 j} v+2 j C^{k-1} v \text { if } j \leqslant 1 / 2 k .
\end{aligned}
$$

Therefore $C^{*} C^{k} v=\beta_{k} C^{k-1} v$ where

$$
\beta_{k}= \begin{cases}k, & k \text { even, } \\ k-1+2 q, & k \text { odd, }\end{cases}
$$

Let $K_{1}$ be the closure of $M$, and let $K_{2}=M^{\perp}$ so that $K=K_{1} \oplus K_{2}$. Let $v_{j}=$ $\alpha_{j} C^{j} v$, where $\alpha_{j}$ is chosen to make $v_{j}$ a unit vector, i.e.

$$
\alpha_{0}=\|v\|^{-1}, \quad \alpha_{j}=\|v\|^{-1}\left[\prod_{k=1}^{j} \beta_{k}\right]^{-1 / 2}, \quad j \geqslant 1 .
$$

Then $v_{j} \in K_{1}$, and if $j>k$,

$$
\left\langle v_{j}, v_{k}\right\rangle=\alpha_{j} \alpha_{k}\left\langle C^{j} v, C^{k} v\right\rangle=\alpha_{j} \alpha_{k}\left\langle C^{j-k-1} v, C^{*(k+1)} C^{k} v\right\rangle=0,
$$

since $C^{*(k+1)} C^{k} v$ is proportional to $C^{*} v$. Therefore $\left\langle v_{j}, v_{k}\right\rangle=\delta_{j k}$. Simple calculations yield 


$$
\begin{aligned}
& C v_{j}=\sqrt{\beta_{j+1}} v_{j+1} ; \quad C^{*} v_{j}=\sqrt{\beta_{j}} v_{j-1}, \quad \text { if } j \geqslant 1 ; \\
& C^{*} C v_{j}=\beta_{j+1} v_{j} ; \quad C C^{*} v_{j}=\beta_{j} v_{j}, \quad \text { if } j \geqslant 1 .
\end{aligned}
$$

Let $T=\sqrt{C^{*} C} . T^{2} v_{j}=\beta_{j+1} v_{j}$ so $T v_{j}=\sqrt{\beta_{j+1}} v_{j}$. If $P_{j}$ projects on the one-dimensional space spanned by $v_{j}, P_{j}$ commutes with all of the spectral projections of $T$. Therefore $P=\Sigma_{j=1}^{\infty} P_{j}$ does also. Thus $P T \subseteq T P$, so if $w \in$ $\operatorname{Dom}(T)$ then $P w \in \operatorname{Dom}(T)$. Since $\operatorname{Dom}(T)=\operatorname{Dom}(C)$, if $w \in \operatorname{Dom}(C)$ then $P w$ $\in \operatorname{Dom}(C) . P$ projects onto $K_{1}$ so

$$
\operatorname{Dom}(C)=\operatorname{Dom}(C) \cap K_{1} \oplus \operatorname{Dom}(C) \cap K_{2} .
$$

A similar argument applied to $S=\sqrt{C C^{*}}$ yields

$$
\operatorname{Dom}\left(C^{*}\right)=\operatorname{Dom}\left(C^{*}\right) \cap K_{1} \oplus \operatorname{Dom}\left(C^{*}\right) \cap K_{2} .
$$

It is now a simple matter to prove the following four statements:

(i) $w \in \operatorname{Dom}(C) \cap K_{2}$ implies $C w \in K_{2}$,

(ii) $w \in \operatorname{Dom}\left(C^{*}\right) \cap K_{2}$ implies $C^{*} w \in K_{2}$,

(iii) $w \in \operatorname{Dom}(C) \cap K_{1}$ implies $C w \in K_{1}$,

(iv) $w \in \operatorname{Dom}\left(C^{*}\right) \cap K_{1}$ implies $C^{*} w \in K_{1}$.

We will prove (ii) and (iii). (i) and (iv) are done similarly. If $w \in \operatorname{Dom}\left(C^{*}\right) \cap K_{2}$ and $x \in M,\left\langle C^{*} w, x\right\rangle=\langle w, C x\rangle=0$ so $C^{*} w \in M^{\perp}=K_{2}$ which gives (ii). If $w \in \operatorname{Dom}(C) \cap K_{1}$ and $x \in \operatorname{Dom}\left(C^{*}\right) \cap K_{2},\langle C w, x\rangle=\left\langle w, C^{*} x\right\rangle=0$ by (ii) so $C w \in\left(\operatorname{Dom}\left(C^{*}\right) \cap K_{2}\right)^{\perp}=K_{2}^{\perp}=K_{1}$ since $\operatorname{Dom}\left(C^{*}\right) \cap K_{2}$ is dense in $K_{2}$ by (5). For $i=1,2$ define $C_{i}$, a linear operator on $K_{i}$, as the restriction of $C$ to $K_{i}$. We will next show that $C_{i}^{*}$ is the restriction of $C^{*}$ to $K_{i}$.

Let $w \in \operatorname{Dom}\left(C^{*}\right) \cap K_{i}$. If $x \in \operatorname{Dom}\left(C_{i}\right)$ then

$$
\left\langle C_{i} x, w\right\rangle=\langle C x, w\rangle=\left\langle x, C^{*} w\right\rangle,
$$

so $w \in \operatorname{Dom}\left(C_{i}^{*}\right)$ and $C_{i}^{*} w=C^{*} w$. Thus $\left.C^{*}\right|_{K_{i}} \subseteq C_{i}^{*}$. Now assume $w \in \operatorname{Dom}\left(C_{i}^{*}\right)$. If $x \in \operatorname{Dom}(C), x=x_{1}+x_{2}, x_{1} \in \operatorname{Dom}(C) \cap K_{1}, x_{2} \in \operatorname{Dom}(C) \cap K_{2}$.

$$
\begin{aligned}
\langle C x, w\rangle & =\left\langle C x_{1}, w\right\rangle+\left\langle C x_{2}, w\right\rangle=\left\langle C x_{i}, w\right\rangle=\left\langle C_{i} x_{i}, w\right\rangle \\
& =\left\langle x_{i}, C_{i}^{*} w\right\rangle=\left\langle x_{1}, C_{i}^{*} w\right\rangle+\left\langle x_{2}, C_{i}^{*} w\right\rangle=\left\langle x, C_{i}^{*} w\right\rangle,
\end{aligned}
$$

so $w \in \operatorname{Dom}\left(C^{*}\right)$ and $C^{*} w=C_{i}^{*} w$. Thus $\left.C_{i}^{*} \subseteq C^{*}\right|_{K_{i}}$. This shows that $C=C_{1}$ $\oplus C_{2}$ where $C_{1}$ and $C_{2}$ each satisfy the conditions of the theorem and

$$
C_{1} v_{j}=\sqrt{\beta_{j+1}} v_{j+1}=\gamma_{j} v_{j+1} \text { if } p=2 q .
$$

By transfinite induction, we may write

$$
K=\left(\sum_{\mu} \oplus K_{\mu}\right) \oplus K_{0}, \quad C=\left(\sum_{\mu} \oplus C_{\mu}\right) \oplus C_{0},
$$


where for each $\mu,\left\{C_{\mu}, K_{\mu}\right\}$ is a free paraboson field and $\left\{C_{0}, K_{0}\right\}$ satisfies the conditions of the theorem but $1 / 2\left(C_{0} C_{0}^{*}+C_{0}^{*} C_{0}\right)$ has no point spectrum.

The theorem is now a consequence of the following statement: If $\sigma_{d}(n)=$ $\varnothing$ then $\{C, K\}$ is reducible. To see that this is true suppose $\sigma_{d}(n)=\varnothing$. If $\Delta$ is a bounded set of real numbers and $w \in E(\Delta) K$, then $w \in \operatorname{Dom}(n)$ and

$$
\begin{aligned}
\langle C w, C w\rangle+\left\langle C^{*} w, C^{*} w\right\rangle & =\left\langle w, C^{*} C w\right\rangle+\left\langle w, C C^{*} w\right\rangle \\
& =\langle w, 2 n w\rangle \leqslant 2\|\Delta\|\|w\|^{2}
\end{aligned}
$$

where $\|\Delta\|=\sup \Delta$. Thus, $n, C$ and $C^{*}$ are bounded on $E(\Delta) K$. Now assume $\Delta$ is a bounded open interval, $\Delta=(a, b)$, and suppose $E(\Delta) w=w$. We will show that

$$
\begin{gathered}
E(\Delta+1) C w=C w, \\
E(\Delta-1) C^{*} w=C^{*} w
\end{gathered}
$$

where $\Delta+k=\{d+k: d \in \Delta\}$

Let $k$ be a nonnegative integer and let $\delta=(b-a) / 2 k$. Assume $k$ is sufficiently large so that $\delta<1$.

Let $\Delta_{j}=(a+2 j \delta, a+2(j+1) \delta)$ for $j=0,1, \ldots, k-1$. Let $w_{j}=$ $E\left(\Delta_{j}\right) w$ so $w=\Sigma_{j=0}^{k-1} w_{j}$ and $E\left(\Delta_{j}\right) w_{j}=w_{j}$. Let $\theta_{j}=a+2(j+1 / 2) \delta$, the midpoint of $\Delta_{j}$, and let

$$
\begin{aligned}
z & =n w-\sum_{j=0}^{k-1} \theta_{j} w_{j}=\sum_{j=0}^{k-1}\left(n-\theta_{j}\right) w_{j} . \\
\|z\|^{2} & =\left\|\sum_{j=0}^{k-1}\left(n-\theta_{j}\right) w_{j}\right\|^{2}=\sum_{j=0}^{k-1}\left\|\left(n-\theta_{j}\right) w_{j}\right\|^{2} \\
& \leqslant \delta^{2} \sum_{j=0}^{k-1}\left\|w_{j}\right\|^{2}=\delta^{2}\|w\|^{2}
\end{aligned}
$$

where we have used that the $w_{j}$ 's are orthogonal.

Since $E(\Delta) z=z$,

$$
\begin{aligned}
\|C z\|^{2} & \leqslant 2\|\Delta\|\|z\|^{2} \leqslant 2\|\Delta\| \delta^{2}\|w\|^{2} . \\
C z & =\sum_{j=0}^{k-1} C\left(n-\theta_{j}\right) w_{j}=\sum_{j=0}^{k-1}\left(n-\theta_{j}-1\right) C w_{j} .
\end{aligned}
$$

This is a sum of orthogonal terms since

$$
\left\langle C\left(n-\theta_{j}\right) w_{j}, C\left(n-\theta_{j^{\prime}}\right) w_{j^{\prime}}\right\rangle=\left\langle\left(n-\theta_{j}\right) w_{j}, C^{*} C\left(n-\theta_{j^{\prime}}\right) w_{j^{\prime}}\right\rangle
$$

and $\left(n-\theta_{j}\right) w_{j} \in E\left(\Delta_{j}\right) K$ while $C^{*} C\left(n-\theta_{j^{\prime}}\right) w_{j^{\prime}} \in E\left(\Delta_{j^{\prime}}\right) K$ since $C^{*} C$ commutes with $n$. 


$$
\sum_{j=0}^{k-1}\left\|\left(n-\theta_{j}-1\right) C w_{j}\right\|^{2}=\|C z\|^{2} \leqslant 2\|\Delta\| \delta^{2}\|w\|^{2}
$$

Let $\epsilon_{j}=\left\|\left(n-\theta_{j}-1\right) C w_{j}\right\|$, so $\Sigma_{j=0}^{k-1} \epsilon_{j}^{2} \leqslant 2\|\Delta\| \delta^{2}\|w\|^{2}$. Let $\Delta_{j}^{0}=\left(\theta_{j}-\sqrt[4]{\delta}, \theta_{j}+\right.$ $\sqrt[4]{\delta})$ so $\Delta_{j} \subseteq \Delta_{j}^{0}$. Let $y_{j}=E\left(\Delta_{j}^{0}+1\right) C w_{j}-C w_{j}$. Then

$$
\begin{aligned}
\left\|y_{j}\right\|^{2} & =\left\|E\left(\left(\Delta_{j}^{0}+1\right)^{\prime}\right) C w_{j}\right\|^{2} \\
& \leqslant\left\|E\left(\left(\Delta_{j}^{0}+1\right)^{\prime}\right)\left(\frac{n-\theta_{j}-1}{\sqrt[4]{\delta}}\right) C w_{j}\right\|^{2} \\
& \leqslant\left\|\left(\frac{n-\theta_{j}-1}{\sqrt[4]{\delta}}\right) C w_{j}\right\|^{2} \leqslant \frac{\epsilon_{j}^{2}}{\sqrt{\delta}}
\end{aligned}
$$

where ' denotes the complement. Let $\Delta^{0}=\bigcup_{j=0}^{k-1} \Delta_{j}^{0}$ and $y_{j}^{0}=E\left(\Delta^{0}+1\right) C w_{j}-$ $C w_{j}$. Then $\left\|y_{j}^{0}\right\|^{2} \leqslant\left\|y_{j}\right\|^{2} \leqslant \epsilon_{j}^{2} \sqrt{\delta}$. Let $y=\sum_{j=0}^{k-1} y_{j}^{0}=E\left(\Delta^{0}+1\right) C w-C w$.

$$
\|y\|^{2} \leqslant k \sum_{j=0}^{k-1}\left\|y_{j}^{0}\right\|^{2} \leqslant \frac{k}{\sqrt{\delta}} \sum_{j=0}^{k-1} \epsilon_{j}^{2} \leqslant\|\Delta\|^{2}\|w\|^{2} \sqrt{\delta} .
$$

As $k \rightarrow \infty, \delta \rightarrow 0, \Delta^{0}+1 \rightarrow \Delta+1$ so $\|y\| \rightarrow 0, E\left(\Delta^{0}+1\right) C w \rightarrow C w$, $E\left(\Delta^{0}+1\right) \rightarrow E(\Delta+1)$ strongly and thus $E(\Delta+1) C w=C w$. This establishes (6).

To see that (7) holds, let $\Delta_{1}$ be a bounded interval disjoint from $\Delta-1$ and assume $E\left(\Delta_{1}\right) y=y$,

$$
\left\langle C^{*} w, y\right\rangle=\langle w, C y\rangle=\left\langle E(\Delta) w, E\left(\Delta_{1}+1\right) C y\right\rangle=0 .
$$

Therefore, $C^{*} w \in\left(E\left((\Delta-1)^{\prime}\right) K\right)^{\perp}$ so $E(\Delta-1) C^{*} w=C^{*} w$.

Let $q=\inf \sigma(n)$. Choose $\epsilon, 0<\epsilon<1$, such that $E((q+\epsilon, q+1)) \neq 0$. Let $\Delta=(q, q+\epsilon)$. Let $M=\left\{w \in K: \Sigma_{k=0}^{m} E(\Delta+k) w=w\right.$ for some $\left.m\right\} \subseteq D$. Let $w \in M$. Define $w_{k}=E(\Delta+k) w$ so $w=\Sigma_{k=0}^{m} w_{k} . C w=\Sigma_{k=0}^{m} C w_{k}$ and so $C w \in M . C^{*} w=\sum_{k=0}^{m} C^{*} w_{k}$ and $E(\Delta+k-1) C^{*} w_{k}=C^{*} w_{k}$. Since $E(\Delta-1)=0$, $C^{*} w \in M$. Thus $C M \subseteq M$ and $C^{*} M \subseteq M$. Let $K_{1}$ be the closure of $M, K_{2}=M^{\perp}$ and $P=\Sigma_{k=0}^{\infty} E(\Delta+\bar{k})$ so that $P$ projects on $K_{1}$ and is a spectral projection of $n$. Since $T=\sqrt{C^{*} C}$ and $S=\sqrt{C C^{*}}$ commute with $n, P T \subseteq T P$ and $P S \subseteq S P$ so, as before,

$$
\begin{gathered}
\operatorname{Dom}(C)=\operatorname{Dom}(C) \cap K_{1} \oplus \operatorname{Dom}(C) \cap K_{2}, \\
\operatorname{Dom}\left(C^{*}\right)=\operatorname{Dom}\left(C^{*}\right) \cap K_{1} \oplus \operatorname{Dom}\left(C^{*}\right) \cap K_{2},
\end{gathered}
$$

and $C=C_{1} \oplus C_{2} . K_{1} \neq\{0\}$ since $E(\Delta) \neq 0$ by the definition of $q . K_{2} \neq\{0\}$ since $E((q+\epsilon, q+1)) \neq 0$. Thus $\{C, K\}$ is reducible. 
This completes the proof of the theorem.

COROLlARY. There are no bounded representations of Green's paraboson relations.

Proof. If $C$ were any bounded creator from a representation of the paraboson relations, it would have to satisfy (2). $C C^{*}$ and $C^{*} C$ commute since their commutator is formally zero and they are bounded. We may assume that $0 \notin$ $\sigma_{d}(n)$ since $C$ and $C^{*}$ act trivially on $E(\{0\})$. Let $M$ be an even integer greater than sup $\sigma(n)$. By (6), $C^{M}=0$. Choose $q \in \sigma(n)$ such that $q>0$ and $q-1 / 2<$ inf $\sigma(n)$. Let $\Delta=(q-1 / 2, q+1 / 2)$. If $E(\Delta) w=w$ then $C^{*} w=0$ since $E(\Delta-1) C^{*} w$ $=C^{*} w$ and $E(\Delta-1)=0$. Suppose $E(\Delta) w=w$ and $\|w\|=1$. By (3),

$$
C^{*} C^{k} w= \begin{cases}k C^{k-1} w, & k \text { even, } \\ (k-1) C^{k-1} w+C^{k-1} C^{*} C w, & k \text { odd. }\end{cases}
$$

Let $v=n w-q w . C^{*} C w=2 n w=2 q w+2 v$.

$$
\begin{aligned}
C^{*} C^{k} w & = \begin{cases}k C^{k-1} w, & k \text { even, } \\
(k-1+2 q) C^{k-1} w+2 C^{k-1} v, & k \text { odd. }\end{cases} \\
\left\|C^{k} w\right\|^{2} & =\left\langle C^{k} w, C^{k} w\right\rangle \\
& =\left\langle C^{k-1} w, C^{*} C^{k} w\right\rangle \\
& = \begin{cases}k\left\|C^{k-1} w\right\|^{2}, & k \text { even, } \\
(k-1+2 q)\left\|C^{k-1} w\right\|^{2}+2\left\langle C^{k-1} w, C^{k-1} v\right\rangle, & k \text { odd. }\end{cases}
\end{aligned}
$$

If $k$ is even and $2 \leqslant k \leqslant M$,

$$
\begin{aligned}
\left\|C^{k} w\right\|^{2} & =k(k-2+2 q)\left\|C^{k-2} w\right\|^{2}+2 k\left\langle C^{k-2} w, C^{k-2} v\right\rangle \\
& \geqslant 2 q k\left\|C^{k-2} w\right\|^{2}-2 M\|C\|^{2 k-4}\|v\| .
\end{aligned}
$$

Since $\|C\|^{2}=\left\|C^{*} C\right\| \leqslant 2\|n\| \leqslant 2 M$,

$$
\begin{aligned}
\left\|C^{k} w\right\|^{2} & \geqslant 2 q k\left\|C^{k-2} w\right\|^{2}-(2 M)^{k-1}\|v\| \\
& \geqslant 2 q k\left(2 q(k-2)\left\|C^{k-4} w\right\|^{2}-(2 M)^{k-3}\|v\|\right)-(2 M)^{k-1}\|v\| \\
& \geqslant(2 q)^{k / 2} k(k-2) \cdots(2)\|w\|^{2}-\|v\|\left((2 M)^{k-1}+q(2 M)^{k-2}+\cdots\right) . \\
\left\|C^{M} w\right\|^{2} & \geqslant(2 q)^{M / 2} M(M-2) \cdots(2)-\|v\| P(M)
\end{aligned}
$$


where $P(M)$ is some fixed positive function of $M$. Thus

$$
\|v\| \geqslant \frac{(2 q)^{M / 2} M(M-2) \cdots(2)}{P(M)}=Q .
$$

We can now find a sequence, $w_{j}$, with $E(\Delta) w_{j}=w_{j},\left\|w_{j}\right\|=1$ and $n w_{j} \rightarrow$ $q w_{j}$. We then have $\left\|v_{j}\right\| \geqslant Q$ but $v_{j} \rightarrow 0$. This contradiction completes the proof of the corollary.

If $H$ is a complex Hilbert space, a quantum field over $H$ is a collection $\{K, C, \Gamma, v\}$ where $K$ is a complex Hilbert space, $C$ is a complex linear function from $H$ into the closed densely defined operators on $K, \Gamma$ is a continuous representation of the unitary group of $H$ on $K$ and $v$ is a vector in $K$ which is cyclic for the algebra generated by the $C(z)$ and $C^{*}(z)$ such that

$$
\Gamma(U) C(z) \Gamma(U)^{-1}=C(U z), \quad \Gamma(U) v=v,
$$

for all unitaries $U$ on $H$ and all $z \in H$. The quantum field is said to be positive (or have positive energy) if when $A$ is a nonnegative self-adjoint operator on $H$, the operator $d \Gamma(A)$ defined by $\Gamma\left(e^{i t A}\right)=e^{i t d \Gamma}(A)$ is also nonnegative. In this case we write $d \Gamma \geqslant 0$. The standard parafermion fields are examples of positive energy quantum fields [7] as are the free boson fields [1, Corollary 2, Theorem 4, Theorem 8]. The one-dimensional free paraboson field can also be given this structure as follows.

Let $H$ be a one-dimensional Hilbert space. $H$ is just the field of complex numbers. Let $\{C, K\}$ be the one-dimensional free paraboson field. For $\alpha \in H$, define $C(\alpha)=\alpha C$. A representation $\Gamma$ of the unitary group $\mathrm{U}(H)$ of $H$ on $K$ can then be defined which intertwines with $C(\alpha) . \mathrm{U}(H)$ is just the set of complex numbers with absolute value 1 . Define $\Gamma(\beta)$ for $\beta \in \mathrm{U}(H)$ by $\Gamma(\beta) e_{j}=\beta^{j} e_{j} . \quad \Gamma$ is then a representation and $\Gamma(\beta) C(\alpha) \Gamma(\beta)^{-1} e_{j}=C(\beta \alpha) e_{j}$. If we define $v=e_{0}$, we get $\Gamma(\beta) v=v$. For any self-adjoint operator $A$ on $H, A$ is just multiplication by some real number $a$ and $d \Gamma(A) e_{j}=j a e_{j}$. Hence, $d \Gamma(A) \geqslant 0$ when $A \geqslant 0$. This implies that $\{K, C, \Gamma, v\}$ is a positive quantum field over $H$.

The order of the paraboson field can be any nonnegative number $p$. If $p$ is an integer then the field can be obtained from the skew product of $p$ free boson fields by Green's ansatz [3, p. 1157]. When $p$ is not an integer the free paraboson field of order $p$ cannot be obtained from boson fields. This gives the first example of a positive quantum field not derivable from boson or fermion fields for which the creation operators satisfy simple relations.

\section{BIBLIOGRAPHY}

1. J. M. Cook, The mathematics of second quantization, Trans. Amer. Math. Soc. 74 (1953), 222-245. MR 14, 825.

2. H. S. Green, A generalized method of field quantization, Phys. Rev. (2) 90 (1953), 270-273. MR 14, 1046. 
3. O. W. Greenberg and A. M. L. Messiah, Selection rules for parafields and the absence of para particles in nature, Phys. Rev. (2) 138 (1965), B1155-1167. MR 32 \#5108.

4. E. Nelson, Analytic vectors, Ann. of Math (2) 70 (1959), 572-615. MR 21 \#5901.

5. C. R. Putnam, Remarks on certain operators of quantum field theory, J. London Math. Soc. 29 (1954), 350-354. MR 16, 146.

6. - Commutation properties of Hilbert space operators and related topics, Ergebnisse der Mathematik und ihrer Grenzgebiete, Band 36, Springer-Verlag, New York, 1967. MR 36 \#707.

7. S. Robbins, Uniqueness of the positive energy parafermion field, Comm. Math. Phys. 38 (1974), 111-118.

8. H. G. Tillmann, Zur Eindeutigkeit der Lösungen der quantenmechanischen Vertauschungsrelationen, Acta Sci. Math. (Szeged) 24 (1963), 258-270. MR 28 \#1878.

DEPARTMENT OF MATHEMATICS, MASSACHUSETTS INSTITUTE OF TECHNOLOGY, CAMBRIDGE, MASSACHUSETTS 02139

Current address: Division of Mathematics, Computer Science and System Design, University of Texas at San Antonio, San Antonio, Texas 78284 